\title{
Método para medir la inmadurez neuronal en el estrabismo congénito
}

\author{
Method for measuring neuronal immaturity in congenital strabismus
}

\author{
Martín Gallegos-Duarte' ${ }^{1}$ Jorge Domingo Mendiola-Santibañez ${ }^{2}$
}

Para citar este artículo: Gallegos-Duarte M, Mendiola-Santibañez JD. Método para medir la inmadurez neuronal en el estrabismo congénito. Ustasalud 2018; 17: 7-14

\begin{abstract}
Licencia Creative Commons (c) (1) $(9)$ lo tanto, los lectores pueden acceder libremente a los artículos en su formato .pdf, igualmente podrán descargarlos y difundirlos; sin embargo no podrán modificarlos o alterarlos, adicionalmente se debe reconocer la autoría de las personas que figuran en las publicaciones, pero estas no podrán ser comercializadas.
\end{abstract}

\section{RESUMEN}

Objetivo: Determinar el grado de inmadurez neuronal en el estrabismo esencial mediante análisis Voxel (granulometría y FreeSurfer) de la corteza cerebral.

Material y métodos: Se realizó un estudio piloto, prospectivo, transversal, observacional para analizar la densidad granulométrica y el grosor cortical de los cerebros de seis niños de siete años de edad, agrupados de la siguiente manera: dos niños sanos como grupo control (GC), dos niños con esotropia congénita (ET), dos niños con exotropia disociada (XTD), y un niño de 2 años de edad con leucomalacia (LM). Los resultados fueron comparados para su análisis.

Resultados: El GC mostró la mayor cantidad de elementos granulométricos en la sustancia blanca, mientas que el caso de LM mostró la menor cantidad así como un aumento de volumen de estos; los casos de estrabismo ocuparon posiciones intermedias entre estos dos parámetros. Mediante FreeSurfer se identificó en el grupo problema un aumento del espesor en la corteza estriada y una disminución en los lóbulos temporales.

Conclusión: La inmadurez neuronal está representada por la disminución de la densidad de la sustancia blanca, el aumento en la sustancia gris, la disminución del grosor cortical en los lóbulos temporales y el aumento de grosor cortical en lóbulos occipitales. Existe una relación cuantitativa entre la cantidad, calidad, proporción y distribución de los elementos granulométricos de la sustancia gris y blanca de la corteza cerebral que permite establecer de una manera objetiva la inmadurez neuronal mediante análisis Voxel.

Palabras clave: Corteza cerebral, estrabismo, neuroimagen, sustancia gris, sustancia blanca, Voxel.

\section{ABSTRACT}

Objective: To determine the degree of neuronal immaturity in the essential strabismus by Voxel analysis (particle size and FreeSurfer) of the cerebral cortex.

Methods: We conducted a pilot study, prospective, transversal, and observational to analyze the density particle and cortical thickness of the brains of six children seven years of age, grouped as follows: Two healthy children as a control group (GC), two children With Congenital esotropia (ET), two children with dissociated Exotropia (XTD), and a 2-year-old child with Periventricular (LM). The results were compared for analysis.

Results: The GC showed the most granulometric elements in the white substance, while the case of LM showed the least amount as well as an increase in volume of these; the cases of strabismus occupied intermediate positions between these two parameters. By FreeSurfer, an increase in the thickness of the striated cortex and a decrease in the temporal lobes were identified in the problem group.

Conclusion: The neuronal immaturity is represented by the decrease of the density of the white substance, the increase in the gray substance, the decrease of the cortical thickness in the temporal lobes and the increase of cortical thickness in occipital lobes. There is a quantitative relation between the quantity, quality, proportion and distribution of the granulometric elements of the gray and white substance of the cerebral cortex that allows establishing in an objective way the neuronal immaturity by means of Voxel analysis.

Keywords: Cerebral cortex, strabismus, neuroimaging, gray substance, white substance, Voxel.

Recibido para publicación:

09 de julio 2018

Aceptado para publicación

04 de diciembre 2018 


\section{INTRODUCCIÓN}

El estrabismo congénito o esencial (EC) es básicamente una enfermedad neurológica que se manifiesta como una desviación ocular patológica. Esta desviación puede ser hacia adentro (esotropia) o bien hacia afuera (exotropia) y puede presentar además movimientos disociados ${ }^{1}$. Se considera que el origen del EC es multifactorial y, es la inmadurez neuronal, uno de los factores que más se ha relacionado con el origen esta enfermedad ${ }^{1-4}$.

Los movimientos disociados en el EC indican la presencia de alteraciones en el desarrollo de los circuitos de interconexión corticales y cortico-subcorticales $^{1,3}$. Estas alteraciones han sido investigadas mediante métodos neurofuncionales y morfométricos de alto discernimiento temporal y espacial ${ }^{1,3-5}$.

El EC se presenta con una sinología clínica fácil de sistematizar, capaz de correlacionarse con los estudios de neuroimagen ${ }^{3,4,6}$. Entre las técnicas de discernimiento espacial fino que han permitido sondear la morfología cortical in vivo de niños estrábicos, está el análisis Voxel ${ }^{7,8}$. Este consiste en identificar los Pixel (en una imagen bidimensional, constituye la unidad mínima formadora de imagen o picture elements) y los Voxel (en una imagen tridimensional, constituye la unidad básica de una matriz tridimensional de datos o volumen elements) obtenidos a partir de imágenes positivas tridimensionales de resonancia magnética o RMI T1 de 3D 8 .

Existen variantes de la técnica Voxel para la reconstrucción de imágenes; una de ellas, denominada FreeSurfer, es un conjunto de herramientas automatizadas que permiten la superposición de pixeles hasta lograr la reconstrucción de mapas digitalizados y superficies muy precisas que se obtienen a partir de las complejas y heterogéneas imágenes de los surcos y grosores corticales del cerebro $^{7,8}$. Esta técnica se ha empleado para identificar cambios en la distribución del volumen de la sustancia gris en la corteza cerebral de pacientes con ambliopía ${ }^{7}$, así como para identificar cambios morfométricos en la corteza cerebral de pacientes estrábicos ${ }^{1,8}$.

Mientras que la madurez se entiende como el final armónico y pleno de un proceso para un tiempo determinado 9 , por su parte, la inmadurez representa una dificultad para alcanzar el completo desarrollo del sistema nervioso central ${ }^{10}$.

Un inadecuado desarrollo de los circuitos corticales y cortico-subcorticales por disturbios en las inter-neuronas y en las células de proyección, debido a daño lesional ya sea por asfixia o hipoxia, puede alterar la sustancia blanca y con ello comprometer la madurez neuronal ${ }^{11,12}$.

Estudios hechos en ratas han demostrado que ante el estrés oxidativo, la respuesta de los vasos corticales induce degeneración vascular, posteriormente una disminución de la masa neuronal y finalmente una disfunción cortical, lo que implica algún grado de inmadurez o retraso neuronal en las crías ${ }^{13}$.

Lo anterior significa que el proceso madurativo puede ser alterado por cambios en la concentración de oxígeno y, que estos cambios, de alguna manera dificultan el correcto desarrollo de los neuroblastos ubicados en las áreas periventriculares ${ }^{14}$, lo que resulta en inmadurez neuronal.

Existen además otras razones para que el proceso de maduración neuronal y visual no culmine de la mejor manera $^{15,16}$, tal y como ocurre cuando existe bajo peso al nacer ${ }^{17}$. Del mismo modo, una alteración en la secuencia del desarrollo neuronal, sea debido a un daño genético $^{14}$, o bien, debido a daño lesional por hipoxia, prematuridad, o isquemia, es capaz de alterar la SB cortical y manifestarse como un estrabismo congénito ${ }^{8,18}$.

El objetivo del estudio piloto fue establecer si la composición granulométrica de las imágenes obtenidas mediante RMI podría ser un parámetro morfométrico confiable para evaluar la inmadurez neuronal.

\section{MATERIALES Y MÉTODOS}

Se hizo un estudio prospectivo y observacional a siete niños, todos ellos mestizos y oriundos de la misma localidad en Querétaro, México; seis de ellos de siete años de edad, fueron agrupados de la siguiente manera: dos niños sanos establecieron el parámetro de madurez (GC), mientras que el cerebro de un niño de dos años con antecedente de leucomalacia (LM) estableció el parámetro de inmadurez neuronal. Fueron 
analizados y comparados los estudios de neuroimagen de corteza visual de cuatro niños con diferentes tipos de estrabismo, dos de ellos manifestaban exotropia disociada (XTD) y dos endotropia congénita (ETC).

Ninguno de los cuatro pacientes con estrabismo presentó evidencia clínica de alteración neurológica concomitante. A todos ellos se les realizó historia oftalmológica completa. Para el análisis de RMI se emplearon dos técnicas Voxel: Una de ellas fue al análisis granulométrico o granulometría y el otro mediante FreeSurfer.

El historial clínico de los pacientes sanos y estrábicos incluyó: exploración oftalmológica, determinación de la agudeza visual, examen oculomotor, pruebas de fusión y clasificación clínica del estrabismo, refracción cicloplégica y examen de fondo de ojo. Los movimientos oculares fueron analizados mediante video. Los pacientes fueron explorados por el mismo observador y bajo la misma rutina de exploración en un ambiente neutro.

Se obtuvieron cortes cerebrales de $1 \mathrm{~mm}$ de espesor mediante RMI T1 de 3D de los cerebros de toda la muestra. Estos cortes se realizaron sin medio de contraste, en pacientes cooperadores y sin medicación alguna. Se seleccionaron y analizaron 17 cortes sagitales de cada lóbulo occipital de cada paciente mediante un equipo y protocolo con las siguientes especificaciones: Equipo Intera Philips 1.0T, Versión 10.6 Secuencia FastFeel Echo (FFE), pesada a T1 en modo 3D, campo de visión (FOV) 230 mm, Real FOV (RFOV) 80\% Grueso de corte (SliceThickness) $1 \mathrm{~mm}$, sin espacio entre cortes, tiempo de eco (TE) 6,9 ms; tiempo de repetición (TR) 25 ms; ángulo de desviación (FA) 30 grados; número de excitaciones (NAS): 1; número de cortes: 120, el equipo y los estudios fueron realizados en el Instituto de Neurobiología de la Universidad Nacional Autónoma de Mexico UNAM, campus Juriquilla.

A partir de estos cortes se desarrollaron dos tipos de estudios morfométricos. En el primero de ellos, las imágenes fueron sometidas a un proceso de identificación por zonas de interés que incluyó el retiro virtual del cráneo, núcleos basales y cerebelo, a fin de analizar la morfometría de las sustancias gris y blanca de ambos lóbulos occipitales como zonas de interés ${ }^{8}$ y se determinó el volumen granulométrico el cual repre- sentó la cantidad de elementos contenidos en el área de interés analizada y fue determinado por medio de aperturas matemáticas progresivas a partir de las cuales fueron filtrados todos los elementos que componen las imágenes obtenidas por medio de RMI. Así, una mayor apertura correspondió a un volumen granulométrico mayor y viceversa. El tamaño exacto de los elementos y la distribución de estos fueron detallados en gráficos.

El segundo método de análisis morfométrico midió el grosor cortical de todos los cerebros mediante el programa de procesamiento de imagen computarizado FreeSurfer.

\section{RESULTADOS}

Se obtuvieron siete estudios de neuroimágenes de la corteza visual: dos de sujetos sanos, dos niños con EC, dos niños con XTD y uno con leucomalacia.

El análisis de grosor cortical mediante FreeSorfer mostró una mayor distribución y densidad de los Voxel en la corteza estriada, así como una disminución del grosor cortical en las regiones temporales de los pacientes con estrabismo, respecto al GC.

El análisis granulométrico de la SB y SG en los cuatro grupos reveló diferencias significativas. En el análisis granulométrico el GC mostró la mayor densidad de la SB y la menor densidad de la SG (Figura 1).

El promedio de densidad granulométrica de la SB fue de 0,06810664 en el GC para ET 0,06476871, 0,06636736 para XTD y 0,034871714 para LM (Figura 1), la densidad granulométrica promedio de la SG del GC fue de 0,00825603, para ET 0,0145374, para XTD 0,01590055, LM 0,01720195 (Cuadro 1).

Se obtuvo un patrón de densidad granulométrica de la sustancia gris uniforme desde el volumen 5 al 17, mientras que en la sustancia blanca destacan los volúmenes 14 y 17 (Figura 2A).

La menor densidad de elementos granulométricos de volumen más grande ocurrió en la sustancia blanca del cerebro con Leucomalacia (Figura 1 y Figura 2B). 


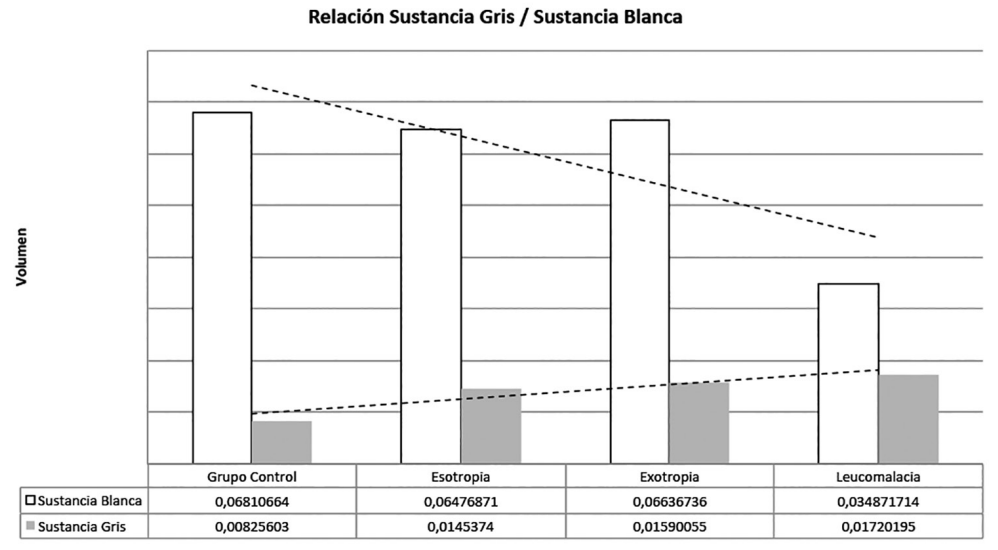

Figura 1. El grupo control (GC) mostró la diferencial mayor entre el volumen de la sustancia gris (columnas color gris) y el de la sustancia blanca (columnas color blanco), mientras que lo contrario sucedió con el cerebro inmaduro; los casos de estrabismo ocuparon posiciones intermedias. Se pudo establecer que un mayor volumen de SB, un menor volumen de SG y una menor diferencia de altura entre las barras grises y blancas, significó madurez; lo contrario sucedió con la inmadurez.

\begin{tabular}{lcc}
\hline & $\begin{array}{c}\text { Densidad granulométrica de } \\
\text { la sustancia blanca }\end{array}$ & $\begin{array}{c}\text { Densidad granulométrica de } \\
\text { la sustancia gris }\end{array}$ \\
\hline Grupo Control & $\uparrow$ & $\downarrow$ \\
Esotropia Congénita & $\downarrow$ & $\downarrow$ \\
Exotropia Disociada & $\uparrow$ & $\uparrow$ \\
Leucomalacia & $\downarrow$ & $\uparrow$ \\
\hline
\end{tabular}

Cuadro 1. Las flechas indican la tendencia en el comportamiento en la densidad granulométrica tanto para sustancia blanca como para sustancia gris de los grupos analizados. El grupo control representa el grado óptimo de "madurez neuronal", mientras el caso de leucomalacia representa el grado mayor de "inmadurez neuronal" en conformidad al análisis granulométrico.

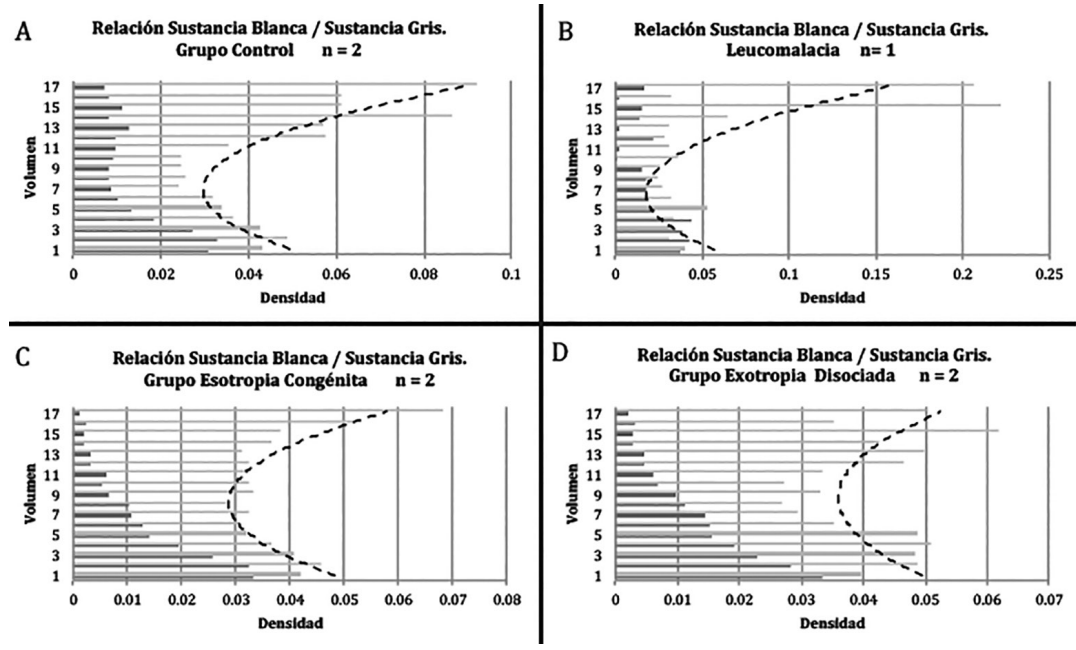

Figura 2. Panel de 4 gráficos identificados con letras mayúsculas que resumen el comportamiento granulométrico de los cuatro grupos analizados. Las barras horizontales indican la relación volumen / densidad de la sustancia gris (barras gris oscuro) y la sustancia blanca (barras en gris claro). La línea punteada pilinominal expresa el comportameinto de la tendencia de la distribución de los elementos granulométircos, además de ser una referencia visual. El eje vertical muestra el volumen (tamaño) de los Voxel determinado por las distintas aperturas matemáticas de los elementos que conforman la imagen; mientras que el eje horizontal muestra la cantidad (densidad) de elementos Voxel del mismo tamaño. 
El grupo ET mostró una disminución relativa de los elementos granulométricos de la sustancia blanca respecto al GC y al grupo XTD, en especial aquellos de volumen 9 y 17 (Figura 1). Mientras que ocurrió un aumento relativo de la densidad de la sustancia gris respecto al GC y una disminución relativa de la sustancia gris respecto al grupo XTD en este grupo (Figura 1 y Figura $2 \mathrm{C}$ ).

El grupo XTD mostró un patrón más parecido al grupo EC que al grupo control y el caso de leucomalacia. Se observó una disminución en los EG de volumen $1,7,9,11$ y 15 en la SB así como una densidad granulométrica de la sustancia cris algo mayor que el grupo de esotropias (Figura 1 y Figura 2 D).

\section{DISCUSIÓN}

Se observaron diferencias básicas entre la forma de presentación del estrabismo, no solo por el comportamiento sensorial y motor sino por los antecedentes familiares y perinatales. Acorde con las historias clínicas, los pacientes con ET tuvieron antecedentes heredofamiliares positivos para la enfermedad, en cambio los pacientes con XTD aunque no poseían antecedentes familiares, presentaron sufrimiento fetal, esto permitió sospechar la posibilidad de que existiera una razón distinta entre ambas formas de presentación, una de ellas debida a alguna causa genética y la otra, debida a sufrimiento fetal.

Los pacientes del grupo XTD manifestaron supresión, ambliopía, y franca asimetría en la manifestación de los movimientos disociados, así como variabilidad en el ángulo de presentación de orto a - 40DP y Desviación Vertical Disociada, todo lo cual indicó un mayor compromiso neuronal. Por su parte, los pacientes con ET manifestaron ambliopía mínima en el ojo no dominante, supresión, desviación constante, uno de ellos de +25 dioptrías prismáticas y el otro de +30 .

Los antecedentes pediátricos y de interconsulta a neurología, no develaron alteración neurológica ni cognitiva subyacente. De igual manera, el rendimiento escolar fue evaluado como regularmente bueno, y el reconocimiento visual por parte de un experto en neuroimagen determinó que las RMI, a excepción del caso con leucomalacia, estaban en límites normales.
Se ha señalado a la inmadurez como uno de los factores causales del estrabismo ${ }^{2,18}$, sin embargo, un asunto es señalar a la inmadurez como una causa vinculada al origen del EC, y otra, establecer con absoluta precisión el grado de inmadurez requerido para que el EC se manifieste en sus variadas formas de presentación.

Para inquirir respecto a las implicaciones que representa la inmadurez cortical, se decidió aplicar la presente metodología al EC, por considerarlo como un modelo biológico con alteraciones corticales ya demostradas ${ }^{1,4,18}$, que resulta sencillo de explorar, calificar y cuantificar de manera precisa en cada una de sus manifestaciones sensoriales y motoras.

Independientemente de que el origen del estrabismo sea lesional o genético, el daño inicial de alguna manera es capaz de modificar la neuroconducción eléctrica. Efectivamente, por medio de estudios neurofuncionales como el Mapeo Cerebral Digitalizado y la Neurometría ya ha sido reportada previamente la presencia de cambios neuroeléctricos en niños con $\mathrm{EC}^{1,4}$.

El objetivo del presente estudio fue investigar, entonces, el sustrato anatómico de donde probablemente surgen dichas alteraciones bioeléctricas, para ello consideramos como ideal utilizar estudios morfométricos finos basados en análisis Voxel, tanto mediante el empleo de técnica granulométrica, como de FreeSurfer.

El estudio piloto estableció el comparativo de los valores matemáticos encontrados mediante neuroimagen en los distintos grupos. Estos valores fueron muy precisos y puntuales, sin embargo, debido el tamaño de la muestra no permite ser tratada estadísticamente.

La idea de emplear estas técnicas, obedece al hecho de que los cambios sutiles en la morfología cortical suelen pasar desapercibidos en los estudios convencionales. En este sentido, el análisis Voxel permitió identificar de forma precisa el volumen y la densidad de los elementos que conforman las imágenes anatómicas y, con ello, se espera conocer mejor respecto de la madurez cortical relacionada con el origen del EC.

Para evaluar objetivamente el grado de madurez cortical y saber si esta se relaciona con la manifestación clínica de la enfermedad, en esta comunicación 
se propuso identificar el comportamiento morfométrico de la corteza cerebral de niños sanos, los cuales fueron considerados a priori, como parámetro de madurez representado por el GC y, se estableció el parámetro de inmadurez, a partir de los datos obtenidos de un cerebro con antecedente de leucomalacia (LM).

A partir de estos dos parámetros, se ubicaron los distintos casos de estrabismo en lo que resultó una escala de valores granulométricos fácilmente expresados en gráficos. La característica distribución de los EG en la SB de cada grupo permitió diferenciar la inmadurez debida a un daño lesional, de aquella de origen genético.

Esto se debe a que la LM siempre es consecuencia de un daño lesional, mientras que los sujetos sanos (GC) no presentan ningún daño. Así, con base en el contraste entre ambos parámetros, resultó sencillo conocer la tendencia en el comportamiento granulométrico de los casos problema y con ello, inferir el tipo de daño que posiblemente originó el estrabismo (Cuadro 1).

Se supuso que en el análisis granulométrico, el GC obtendría la mayor densidad de la SB y así fue. Este grupo obtuvo la menor densidad de la SG, así como el mayor diferencial entre SG y SB; este comportamiento granulométrico estableció el parámetro de madurez neuronal.

Las barras en gris oscuro y claro observadas en los gráficos, representan respectivamente la distribución de la SG y SB, estas mostraron un patrón de densidad en la SG relativamente uniforme desde el volumen 5 al 17, al tiempo que en la SB sobresalieron los volúmenes 14 y 17 .

En el caso de LM se encontró la menor densidad granulométrica para SB y la mayor densidad granulométrica para SG. El caso de LM presentó además, la menor densidad de EG de volumen más grande, esto es, tamaños granulométricos 10,11, 13 y 16, así como una descomposición en el patrón de distribución de los EG de tamaño mayor. El comportamiento de esta tendencia determinó el parámetro granulométrico de inmadurez neuronal.

Las barras mostraron un desorden de los EG, así como una disminución de aquellos elementos de vo- lumen mayor en todos los casos de EC, pero muy especialmente en el caso LM. Esto significó que, desde un punto de vista granulométrico, la inmadurez estuvo representada por una disminución y desorganización de los EG.

Por su parte, la granulometría de la SB del grupo ET manifestó una curva de tendencia polinominal más abierta que el GC, así como una clara disminución de todos los EG de mayor volumen, especialmente aquellos de volumen 9 y 17. Se observó además una disminución relativa de la SG en relación con el grupo de XTD, lo cual podría indicar un origen genético.

Lo anterior resultó congruente con la clínica, ya que el grupo ET presentó, por un lado, el antecedente de tener parientes en primer grado con estrabismo, $y$ por otro, el ángulo de desviación fue constante, con una menor tendencia a la variabilidad, a la asimetría y a la presencia de movimientos disociados, en comparación al grupo XTD, todo lo cual indica clínicamente un menor daño neuronal, en otras palabras, acorde a un criterio de madurez vs inmadurez, el grupo ET presentó una inmadurez relativamente menor que el grupo XTD.

Efectivamente, el grupo XTD exhibió un patrón granulométrico de SB más desorganizado que el GC, así como una disminución en los EG de volumen 1, 7, 9, 11 y 15 en la SB; este grupo manifestó además un aumento relativo de densidad en la SG respecto al grupo ET; en otras palabras, el patrón granulométrico del grupo XTD asumió una tendencia de inmadurez mayor respecto al grupo de ET, y esto es congruente a un daño lesional.

Todos los pacientes estrábicos se ubicaron entre los parámetros establecidos para madurez e inmadurez, en donde los casos de EC se ubicaron en un lugar relativamente más cercano a la madurez que su contraparte, el grupo de XTD.

Los cambios más significativos en la $\mathrm{SB}$ de los casos de estrabismo, ocurrieron en los EG de tamaño medio y pequeño. Naturalmente, se observó una disminución en la cantidad de dichos elementos, así como un desplazamiento hacia la izquierda de la línea 
polinominal en los casos de estrabismo y de LM, lo que indicó que, independientemente del grado, la inmadurez, mostró siempre una disminución de los EG respecto al GC.

Con base en estos resultados, el estudio sugiere que las alteraciones en la proporción y distribución de los EG estarían relacionadas con un déficit en la neuroconducción, por lo que es plausible afirmar, que la inmadurez neuronal, en términos de conectividad, representa un menor desarrollo de las vías de comunicación contenidas en la SB cortical.

Desde un punto de vista biofísico, un acumulo neuronal con una menor cantidad y calidad neuroconductora disminuiría la conductancia, o dicho de otra manera, la inmadurez aumentaría la resistencia bioeléctrica, lo que se traduciría como una densidad mayor de ondas lentas, hiperactividad eléctrica $y$, eventualmente, paroxismos ${ }^{1,3}$. Pues bien, esto es lo que se ha reportado precisamente en pacientes con EC.

Es factible que, debido a la inmadurez neuronal, resulten afectadas en grados y modos distintos las vías de interconexión neuronal, lo que favorecería la heterogeneidad en la expresión clínica del estrabismo congénito.

Por otro lado, el análisis Voxel del espesor cortical mediante FreeSurfer reveló cambios en el grosos cortical en la corteza estriada y en los lóbulos temporales de los pacientes estrábicos, en relación con los sujetos sanos (Figura 3).

Lo anterior sugiere que los pacientes estrábicos requieren un proceso más elaborado para identificar los elementos visuales primarios, como son la identificación de formas, puntos, rayas, sombras, inclinaciones, desenfoque, color y sombras; al tiempo que los lóbulos temporales presentarían cierta dificultad para integrar mediante la memoria de trabajo las imágenes más complejas. Lo anterior es congruente con otra comunicación que reporta disfunciones neuroeléctircas intertemporales ${ }^{3}$.

Las técnicas descritas en el presente artículo indican que el análisis Voxel permite identificar alteraciones en la corteza cerebral relacionadas con inmadurez, la cual ha sido señalada en la literatura como un factor importante en la génesis del estrabismo congénito.
Es plausible considerar la metodología aquí expuesta como una ayuda pronóstica para otras enfermedades donde esté implicada la madurez cortical, así como para inquirir en el origen del EC.

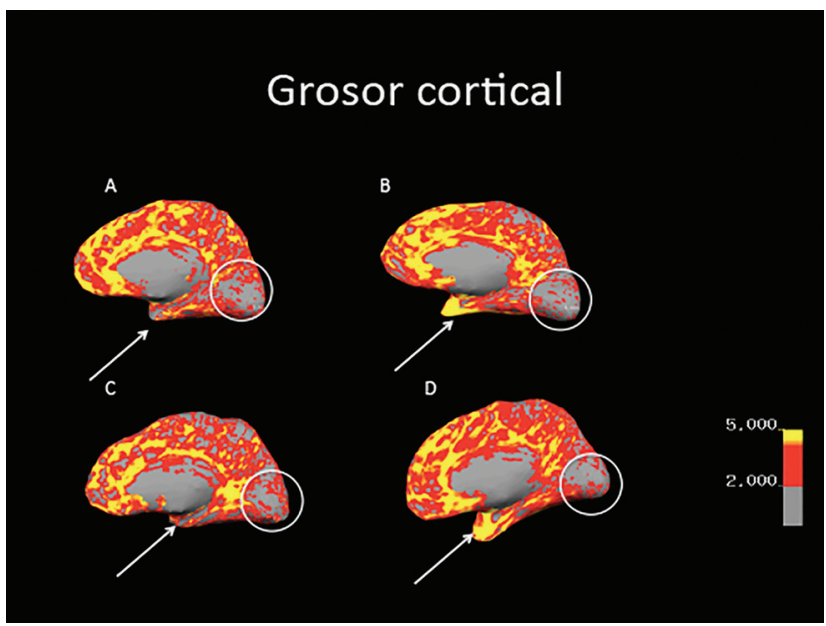

Figura 3. Imagen volumétrica $\mathrm{T} 1$ del hemisferio izquierdo procesada mediante FreeSurfer que muestra la reconstrucción 3D de superficie inflada de un paciente femenino portador de esotropia congenita (A), femenino sano (B), Masculino con exotropia disociada (C), masculino sano (D), todos ellos de 7 años de edad. La escala colorimétrica ubicada en el lado dercho de la imagen indica el grosor cortical en milímetros, en donde el color amarillo representa $5 \mathrm{~mm}$ y el gris menos de $2 \mathrm{~mm}$. Notese un aumento relativo del grosor cortical occipital (en círculos blancos), y el aumento relativo del grosor cortical temporal (con flechas blancas), en los cerebros estrábicos en relación con los sujetos control.

\section{REFERENCIAS}

1. Gallegos-Duarte, Rubio-Chevannier HF, Mendiola-Santibañez JD. Brain Mapping Alterations in Strabismus. In: Diane E. Spinelle. Brain Mapping Alterations. New York: Nova Science Pub Inc; 2011:197-249.

2. Engle EC. Genetic basis of congenital strabismus. Arch Ophtalmol. 2007;125(2):189-95.

3. Gallegos-Duarte M. Alteraciones neuroeléctricas en el estrabismo. Cir Cir 2010;78(3):215-220.

4. Gallegos-Duarte M, Mendiola-Santibáñez JD, Ortiz-Retana JJ; Rubín de Celis B, Vidal-Pineda R, Sigala-Zamora A. Desviación disociada. Un estrabismo de origen cortical. Cir Ciruj. 2007;75(4):241-247. 
5. Gallegos-Duarte M. Estima y origen de la endotropia congénita. Rev Mex Oftalmol 2005;79(1):10-16.

6. Gallegos-Duarte M. Maniobras exploratorias en la endotropia congénita En: Murillo-Murillo C. Estrabismo: México DF. Composición Editorial Láser; 2005:1-18.

7. Mendola JD, Conner IP, Roy A, Chan ST, Schwartz TL, Odom JV, Kwong KK. Voxel-based analysis of MRI detects abnormal visual cortex in children and adults with amblyopia. Human Brain Mapp. 2005;25(2):222-36. DOI: 10.1002/hbm.20109.

8. Mendiola-Santibáñez JD, Gallegos-Duarte M, Ortiz-Retana JJ, López-Campos CE. Segmentación y análisis granulométrico de sustancia blanca y gris para el estudio del estrabismo usando transformaciones morfológicas. Rev Mex Ing Biomed 2007;28(2):92-104.

9. Kostović I, Jovanov-Milosević N. The development of cerebral connections during the first 20-45 weeks' gestation. Semin Fetal Neonatal Med. 2006;11(6):415-22. DOI: 10.1016/j.siny.2006.07.001

10. Kostovic I, Vasung L. Insights from in vitro fetal magnetic resonance imaging of cerebral development. Semin Perinatol. 2009;33(4):220-33. DOI: $10.1053 / \mathrm{j}$. semperi.2009.04.003.

11. Zecevic N, Hu F, Jakovcevski I. Interneurons in the developing human neocortex. Dev Neurobiol. 2011;71(1):1833. DOI: 10.1002/dneu.20812.

12. Alix JJ. The pathophysiology of ischemic injury to developing white matter. Mcgill J Med. 2006; 9(2):134-40.
13. Sirinyan M, Florian Sennlaub F, Dorfman A, Sapieha P, Gobeil F, Hardy P, Lachapelle P, Chemtob S. Hyperoxic exposure leads to nitrative stress and ensuing microvascular degeneration and diminished brain Mass and function in the immature subject. Stroke. 2006;37:2807-15. DOI: 10.1161/01.STR.0000245082.19294.ff.

14. Issaho DC. Genetic Inheritance in Non-Syndromic Infantile Esotropia. Biomed J Sci Tech R10.1136/bjophthalmol-2016-308769es [Internet]. 2018 Feb 1 [cited 2018 Nov 30];2(2). Available from: http://biomedres.us/fulltexts/BJSTR.MS.ID.000716.php.

15. Graziano RM, Leone CR. Frequent ophthalmologic problems and visual development of extremely preterm newborn infants. J Pediatr (Rio J). 2005;81:S95-100.

16. Jacobson L, Rydberg A, Eliasson AC, Kits A, Flodmark O. Visual field function in school-aged children with spastic unilateral cerebral palsy related to different patterns of brain damage. Dev Med Child Neurol. 2010;52(8):e1847. DOI: 0.1111/j.1469-8749.2010.03650.x

17. Stephenson T, Wright S, O'Connor A, Fielder A, Johnson A, Ratib S, Tobin M. Children born weigh ingless than 1701 g: visual and cognitive outcomes at 11-14 years. Arch Dis Child Fetal Neonatal Ed. 2007;92(4):F265-70. DOI: $10.1136 /$ adc.2006.104000.

18. Jeon H, Jung J, Kim H, Yeom JA, Choi H. Strabismus in children with white matter damage of immaturity: MRI correlation. Br J Ophthalmol. 2017;101(4):467-471. DOI: 10.1136/bjophthalmol-2016-308769. 